\title{
The quantum Arnold transformation
}

\author{
V. Aldaya ${ }^{1}$, F. Cossío $^{1}$, J. Guerrero ${ }^{1,2}$ and F.F. López-Ruiz ${ }^{1}$ \\ ${ }^{1}$ Instituto de Astrofísica de Andalucía, CSIC, \\ Apartado Postal 3004, 18080 Granada, Spain \\ ${ }^{2}$ Departamento de Matemática Aplicada, Universidad de Murcia, \\ Campus de Espinardo, 30100 Murcia, Spain. \\ valdaya@iaa.es fcossiop@gmail.es juguerre@um.es flopez@iaa.es
}

\begin{abstract}
By a quantum version of the Arnold transformation of classical mechanics, all quantum dynamical systems whose classical equations of motion are non-homogeneous linear second-order ordinary differential equations, including systems with friction linear in velocity, can be related to the quantum free-particle dynamical system. This transformation provides a basic (Heisenberg-Weyl) algebra of quantum operators, along with well-defined Hermitian operators which can be chosen as evolution-like observables and complete the entire Schrödinger algebra. It also proves to be very helpful in performing certain computations quickly, to obtain, for example, wave functions and closed analytic expressions for time-evolution operators.
\end{abstract}

PACS: 03.65.-w, 02.20.-a, 2.30.Hq

\section{Introduction}

The description of the quantum damped harmonic oscillator by the Caldirola-Kanai model [1, 2], which involves a time-dependent Hamiltonian, has attracted the attention of many authors, as could be considered one of the simplest and paradigmatic examples of dissipative system. In particular, its analysis from the symmetry point of view has proved to be very fruitful. In a purely classical context, the symmetries of the equation of the damped harmonic oscillator with time-dependent parameters were found in 3]. In [4] Cerveró and Villarroel found, for the damped harmonic oscillator, finite-dimensional point symmetry groups for the corresponding Lagrangian (the un-extended Schrödinger group [5]) and the equations of motion $(S L(3, \mathbb{R})$ ) respectively, and an infinite contact one for the set of trajectories of the classical equation. They singled out a "non-conventional" Hamiltonian from those generators of the symmetry, recovering some results from [6, 7].

Some flaws has been associated with the quantum description of the damped harmonic oscillator according to Caldirola-Kanai equation. For instance, it is claimed that uncertainty relations are not preserved under time evolution and could eventually be violated [8, 9], although this inconsistency seems to be associated with a confussion between canonical momentum and "physical" momentum [10].

There exists another interesting approach to the study of the classical damped harmonic oscillator, based on the observation that its classical equation of motion is a special case of the set of linear second-order ordinary differential equations (LSODE for short). In Classical Mechanics the family of solutions of a second-order differential equation corresponding to the motion of a given physical problem is sometimes related to that of a simpler system, considered 
as a toy model, in order to import from it simple general properties which could be hidden in the real problem. Both physical systems should share global properties of the solution manifold, such as topology and symplectic structure. The paradigmatic example is the transformation described by Arnold in [11, which brings any LSODE to the simplest form of the free Galilean particle equation. This transformation turns out to be extremely useful. In particular, it is possible to obtain the symmetry group of a particular instance of LSODE [3], in which the symmetries of the action of the corresponding system can be found as a subgroup [12].

Therefore, it seems natural to try to generalize the Arnold transformation to the quantum level, to be denoted as Quantum Arnold Transformation (QAT), as much insight can be gained in the study of any system classically described by a LSODE and, in particular, the parametric oscillator or some of the systems which present dissipation.

Several partial generalizations can be found in the literature. For example, in [13], Takagi is able to provide a transformation which relates the Schrödinger equation of the harmonic oscillator to that of the free particle, and applies it to simplify the computation of the propagator by making use of the free one. 14 contains a slightly more general version (see formula (33) therein). [15] went a bit further considering the damped harmonic oscillator with constant parameters. None of them mentions the classical Arnold transformation, but it is underlying their reasoning.

Implicitly, a generalization of the Arnold transformation was also contained in [7], the classical version not being referred once more. It will be shown that some of their results formally converge with ours (see Section 4.2), although they put emphasis on another aspects of the problem, such as the analysis of unitarity and energy loss. Mostafazadeh [16] also pursued the idea of "connecting" different quantum physical systems by means of time-dependent unitary transformations, even representing arbitrary time-dependent diffeomorphisms [17. His approach is rather general, but does not fully take advantage of the possibility of connecting with the free particle system and importing its symmetries.

The relevance of generalizing the Arnold transformation is that it can be used to export properties from the free Schrödinger equation to that of the system corresponding to the given classical equation: the complete set of symmetries of the quantum free particle, the Schrödinger group [5, 18], can be realized on the system under study, providing as many conserved quantities as in the free particle. In particular, the generators corresponding to the free position and momentum prove to be good basic quantum operators, constituting a quantization. In addition, the transformation turns out to be extremely useful to compute objects that would otherwise need laborious calculations, such as wave functions, the quantum propagator or the evolution operator.

We shall see that time translations in the non-free system do not belong, in general, to the imported Schrödinger group. This is to be expected, as the energy in this system is not conserved when the classical equation of motion includes a friction term or a time-varying frequency. A deeper analysis of this fact can be found in 19 .

To obtain the QAT, we begin in Section 2 by observing that a generalized version of the classical equation of the damped harmonic oscillator, in which the constant coefficients are promoted to be time-dependent, can be transformed by a local diffeomorphism of position and time, the Arnold transformation, into the equation of motion of the free particle. In Section 3 we generalize this interesting feature from the classical to the quantum theory. The CaldirolaKanai equation for the damped harmonic oscillator is then a particular case of the general one. In Section 4 we illustrate the use of QAT in a couple of simple examples presenting damping, the damped particle and the damped harmonic oscillator. 


\section{Classical Arnold transformation}

Mathematically speaking, the classical Arnold transformation [11] converts any linear secondorder ordinary differential equation (LSODE) into the free Galilean particle equation, that is, $\ddot{\kappa}=0$ in $1+1$ dimensions (we shall limit ourselves to this situation).

From the physical point of view, the Arnold transformation relates the trajectories $x(t)$, with initial conditions $x_{0}$ and $p_{0} \sim \dot{x}_{0}$, solutions of the LSODE, to those trajectories $\kappa(\tau)$ solutions of the free equation with initial conditions $\kappa_{0}$ and $\pi_{0} \sim \dot{\kappa}_{0}$. Either $\left(x_{0}, p_{0}\right)$ or $\left(\kappa_{0}, \pi_{0}\right)$ parametrize the common solution manifold $\mathcal{M}$, and we shall adopt the unified notation $(K, P)$. On this manifold, each physical system is characterized by the corresponding Hamiltonian as a function of $K$ and $P$. The inverse of the corresponding Hamilton-Jacobi transformation then recovers the trajectories $(x(t), \dot{x}(t))$ or $(\kappa(\tau), \dot{\kappa}(\tau))$ out of the $K, P$ variables.

Following a similar notation to that in [3], we give an overview of the Arnold transformation [11. Firstly, let us recall that, given an arbitrary, non-homogeneous LSODE

$$
\ddot{x}+\dot{f} \dot{x}+\omega^{2} x=\Lambda,
$$

where $\dot{x}=\frac{d x}{d t}$ and so on, and $f, \omega$ and $\Lambda$ are arbitrary functions of time $t$, we can apply the transformation

$$
\left\{\begin{array}{l}
t \longrightarrow t \\
x \longrightarrow x+u_{p}
\end{array},\right.
$$

$u_{p}$ being a particular solution of (11). We find that the differential equation above is transformed into

$$
\ddot{x}+\dot{f} \dot{x}+\omega^{2} x=0,
$$

i. e., every non-homogeneous problem is equivalent to a homogeneous one.

The homogeneous Arnold transformation, is a local diffeomorphism which maps the free particle equation of motion into (3):

$$
\left\{\begin{array}{l}
\tau=\frac{u_{1}(t)}{u_{2}(t)} \\
\kappa=\frac{x}{u_{2}(t)}
\end{array} \quad, \quad \ddot{\kappa}=0 \quad \longleftrightarrow \quad \ddot{x}+\dot{f} \dot{x}+\omega^{2} x=0,\right.
$$

where $u_{1}(t)$ and $u_{2}(t)$ are independent solutions of (3). Applying the inverse diffeomorphism to the classical dynamical system (3), we can transform this equation into the free one.

If we include external forces the transformation (4) turns into the general Arnold transformation, that we shall call simply $A$ :

$$
\left\{\begin{array}{l}
\tau=\frac{u_{1}(t)}{u_{2}(t)} \\
\kappa=\frac{x-u_{p}(t)}{u_{2}(t)}
\end{array} \quad, \quad \ddot{\kappa}=0 \quad \longleftrightarrow \quad \ddot{x}+\dot{f} \dot{x}+\omega^{2} x=\Lambda .\right.
$$

This transformation could be understood as passing to coordinates analogous to co-moving spacial coordinate and proper time used in General Relativity, so that the system becomes "free", at least locally.

Indeed this transformation is of local nature in time, in the sense that it is only valid for an open interval in time $t$. In fact, it can be shown that the equivalence in (44) and (5) is true up to a factor $\frac{u_{2}^{2}}{e^{-2 f}}$, so that it holds in the interval where $u_{2}$ does not vanish. This means that the transformation does not take the Euler-Lagrange operator associated with the LSODE itself to 
that of the free system. For this reason it can not be claimed that both physical systems are actually equivalent.

However, Arnold transformation can help to understand the physical system under study. In particular, as pointed in [3], it is possible to identify the set of contact symmetries for (3), and this way to arrive at the results found in [4, which show the sets of symmetries for either the equations or the action from which such equations can be derived.

It should be noted that, due to the general character of the transformation, we could miss the physical identity of position and time when performing such a transformation. But it will be possible to choose appropriate specific solutions $u_{1}, u_{2}$ and $u_{p}$ with suitable initial conditions so that the identity of the variables is maintained 1 . We shall make use of this possibility in going to the quantum version of this transformation.

Finally, having in mind the particular case of dissipative systems, we would like to remark that certain issues of the treatment of these systems are already apparent in the classical domain. For instance, time evolution is not a symplectomorphism, nor preserve the volume of the phase space. Obviously, the Hamiltonian function is not a Noether invariant. This becomes especially manifest and annoying when formulating the quantum theory.

\section{The Quantum Arnold transformation}

As already mentioned in the Introduction, several partial versions of the QAT can be found in the literature. Here we give a generalization that contains, as particular cases, those found in [13, 14, 15, 7].

In bringing Arnold's technique to the quantum world we must be aware, obviously, of the different philosophy of the quantum description and different nature of the equation of motion. The objects and structures that define a quantum system, namely the Hilbert space, the basic observables, the Hamiltonian operator, and the Schrödinger equation must be specified in a way that we are able to identify the same objects at both sides of the transformation.

To this end, it is important to focus, in the free system, only on those operators corresponding to constants of motion, Noether invariants associated with its symmetry, that is, the Schrödinger group (which contains the centrally-extended Galilei group as a subgroup, containing in turn the Heisenberg-Weyl group of translations and non-relativistic boosts). This implies to fix the basic operators (that is to say, quantum operators which realize a unitary and irreducible representation of the common classical Poisson (Heisenberg-Weyl) algebra $\{K, P\}=1$ ) so that they respect the Schrödinger equation, then having constant expectation values and being generators of the basic symmetry 2 (the Heisenberg-Weyl sub-group of the centrally-extended Schrödinger group).

Those basic operators, in this form, are in principle the candidates to be related by a quantum version of the Arnold transformation, so that we shall have the situation as follows: On the one hand, a common Hilbert space $\mathcal{H}$ of wave functions $\Psi(K)\left(L^{2}(\mathbb{R})\right)$, which plays the role of initial values for both the solutions $\phi(x, t) \in \mathcal{H}_{t}$ of the Schrödinger equation relative to the quantum version of our original LSODE and those wave functions, $\varphi(\kappa, \tau) \in \mathcal{H}_{\tau}^{G}$, solutions of the free Schrödinger equation. And, on the other hand, the quantum Arnold transformation $\hat{A}$ relating Schrödinger equations and basic operators. As a crucial consequence, we shall obtain a

\footnotetext{
${ }^{1}$ For his restricted version of the quantum Arnold transformation, Takagi, in [13, suggested that the transformation could be specified by different solutions at different times, in principle avoiding the restriction of locality. However, care must be taken when making use of this freedom to prevent conflict with preserving the identity of the variables when it is desirable.

${ }^{2}$ By "basic symmetry" we understand, in general, those symmetries whose associated Noether invariants are enough to parametrize the classical solution manifold.
} 
realization of the free symmetry on the quantum, non-free system. The following diagram can help to have a picture of the setup:

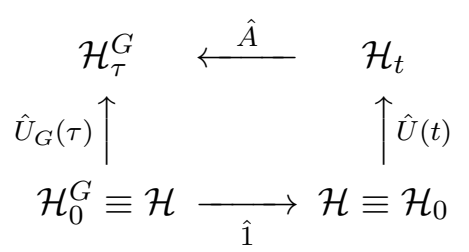

$\mathcal{H}_{\tau}^{G}$ (resp. $\mathcal{H}_{t}$ ) is the Hilbert space of solutions of the free or Galilean (resp. non-free or corresponding to the LSODE) Schrödinger equation, $\hat{U}_{G}(\tau)$ (resp. $\hat{U}(t)$ ) is the free (resp. nonfree) evolution operator and $\hat{1}$ is the identity operator. Here, the Hilbert space $\mathcal{H}$ may be considered as the quantum analogue of the classical solution manifold, $\mathcal{M}$, usually thought of as space of (classical) initial conditions. On $\mathcal{H}$ one must be able to measure all possibles physical observables in much the same way classical observables are characterized as real functions on $\mathcal{M}$, that is, functions whose arguments are constants along classical trajectories (functions of Noether invariants).

The Hamiltonian of the non-free system, not being conserved in general, will not be related to any operator from the free particle. This is to be expected, since it is not a conserved quantity under the evolution of the physical system, neither at classical nor at the quantum level (in the sense that it does not have constant expectation values). It is important to remark that this implies that it is not possible to formulate a time-independent Schrödinger equation.

More specifically, by extending properly the Arnold transformation (or the inverse) to the quantum case, we shall relate the space $\mathcal{H}_{\tau}^{G}$ of solutions of the free Schrödinger equation

$$
i \hbar \frac{\partial \varphi}{\partial \tau}=-\frac{\hbar^{2}}{2 m} \frac{\partial^{2} \varphi}{\partial \kappa^{2}}
$$

with corresponding classical equation

$$
\ddot{\kappa}=0 \text {, }
$$

to that space $\mathcal{H}_{t}$ where the quantum theory of the generic LSODE

$$
\ddot{x}+\dot{f} \dot{x}+\omega^{2} x=\Lambda,
$$

is realized, the quantities $f, \omega$ and $\Lambda$ being, in general, time-dependent.

The classical equation (9) can be derived from a variational principle. We shall consider the Lagrangian

$$
L=e^{f}\left(\frac{1}{2} m \dot{x}^{2}-\frac{1}{2} m \omega^{2} x^{2}+m \Lambda x\right)
$$

as our starting point. The Schrödinger equation can be derived from the corresponding classical Hamiltonian function

$$
H=\frac{p^{2}}{2 m} e^{-f}+\left(\frac{1}{2} m \omega^{2} x^{2}-m \Lambda x\right) e^{f},
$$

according to the standard canonical prescriptions, leading to

$$
i \hbar \frac{\partial \phi}{\partial t}=-\frac{\hbar^{2}}{2 m} e^{-f} \frac{\partial^{2} \phi}{\partial x^{2}}+\left(\frac{1}{2} m \omega^{2} x^{2}-m \Lambda x\right) e^{f} \phi .
$$


For $f$ linear in time, constant $\omega$ and $\Lambda=0$ this equation is commonly known as Caldirola-Kanai equation for the damped harmonic oscillator [1, 2].

Even though both spaces of solutions of (7) and (12), $\mathcal{H}_{\tau}^{G}$ and $\mathcal{H}_{t}$ respectively, will be related, and the basic quantum operators associated with the classical functions $K, P$ realized as welldefined operators both on $\mathcal{H}_{\tau}^{G}$ and $\mathcal{H}_{t}$, we cannot still assure that both physical systems are actually equivalent. In fact, the evolution operator in time $t, i \hbar \frac{\partial}{\partial t}$, does definitely not leave invariant the space of the solutions of (12) in general, nor comes down to the space $\mathcal{H}$, which means that it cannot be realized as an operator function of $\hat{K}, \hat{P}$ (in sharp contrast to $i \hbar \frac{\partial}{\partial \tau}$, which is $\sim \hat{P}^{2}$ ). We shall achieve the construction of well-defined Hermitian evolution-like generators imported from the free system via the inverse quantum Arnold transformation, but their eigenvalues, conserved indeed, do not correspond to the standard energy 3 . These operators close the Schrödinger algebra with the basic operators.

Implicitly, this trick of considering operators different from the Hamiltonian to provide quantum numbers and obtain solutions of the Schrödinger equation as their eigenfunctions has been used extensively. For example, the operator found in [7] to label the energy-loss states, which coincides with the quantum operator $H^{*}$ corresponding to $G_{5}$ in [4, 20], turns out to be a generator of the $S L(2, \mathbb{R})$ subgroup of the Schrödinger group. We give here explicitly the frame in which this can be done: operators from the Schrödinger group can be chosen to play this role upon convenience.

For the sake of simplicity, let us focus on the case with no external forces $\Lambda=0$. The formulas corresponding to $\Lambda \neq 0$ are given in Appendix $\mathrm{A}$.

The generalization of the classical Arnold transformation is obtained by completing (4) with a change of the wave function. Explicitly, the quantum Arnold transformation, valid for every physical system with classical equation of the homogeneous LSODE type, is given by the (local) diffeomorphism:

$$
\left\{\begin{array}{l}
\tau=\frac{u_{1}(t)}{u_{2}(t)} \\
\kappa=\frac{x}{u_{2}(t)} \\
\varphi=\phi \sqrt{u_{2}(t)} e^{-\frac{i}{2} \frac{m}{\hbar} \frac{1}{W(t)} \frac{\dot{u}_{2}(t)}{u_{2}(t)} x^{2}},
\end{array}\right.
$$

where $u_{1}$ and $u_{2}$ satisfy again the classical equation of motion in $(x, t), \dot{u}_{1}=\frac{d u_{1}}{d t}, \dot{u}_{2}=\frac{d u_{2}}{d t}$ and $W(t) \equiv \dot{u}_{1} u_{2}-u_{1} \dot{u}_{2}=e^{-f}$. It is straightforward to check that by this transformation the Schrödinger equation of the free particle is transformed into (12) (with $\Lambda=0$ ) up to a multiplicative factor which depends on the particular choice of the classical solutions $u_{1}$ and $u_{2}$ (partial derivatives must be changed by the classical part of the transformation while wave functions are shifted by the quantum part).

Now, we can impose on $u_{1}$ and $u_{2}$ the condition that they preserve the identity of $\tau$ and $\kappa$, i.e., that $(\kappa, \tau)$ coincide with $(x, t)$ at an initial point $t_{0}$, arbitrarily taken to be $t_{0}=0$ :

$$
u_{1}(0)=0, u_{2}(0)=1, \quad \dot{u}_{1}(0)=1, \dot{u}_{2}(0)=0 .
$$

This fixes a unique form of the diffeomorphism for a given "target" physical system. However, the quantum Arnold transformation would still be valid if solutions $u_{1}$ and $u_{2}$ do not satisfy (14). The price to be paid would then be that the relation in the lower part of the diagram (6) above would no longer be the identity and basic position and momentum operators would then be mixed (see the end of this Section).

\footnotetext{
${ }^{3}$ The construction of a properly defined, Hermitian generator in standard time will take much effort requiring a sound analysis of the symmetry problem in damped systems. This has been done in [19].
} 
Formally, while the classical Arnold transformation in this case is:

$$
\begin{aligned}
A: \mathbb{R} \times T & \longrightarrow \mathbb{R} \times T^{\prime} \\
(x, t) & \longmapsto(\kappa, \tau)=A((x, t))=\left(\frac{x}{u_{2}}, \frac{u_{1}}{u_{2}}\right),
\end{aligned}
$$

where $T$ and $T^{\prime}$ are open intervals of the real line containing $t=0$ and $\tau=0$, respectively, QAT can be written:

$$
\begin{aligned}
\hat{A}: \mathcal{H}_{t} & \longrightarrow \mathcal{H}_{\tau}^{G} \\
\phi(x, t) & \longmapsto \varphi(\kappa, \tau)=\hat{A}(\phi(x, t))=A^{*}\left(\sqrt{u_{2}(t)} e^{-\frac{i}{2} \frac{m}{\hbar} \frac{1}{W(t)} \frac{\dot{u}_{2}(t)}{u_{2}(t)} x^{2}} \phi(x, t)\right),
\end{aligned}
$$

where $A^{*}$ denotes the pullback operation corresponding to $A$.

As already remarked, the basic symmetries of the free system are inherited by the LSODEtype system, as we are now able to transform the infinitesimal generators of translations (the Galilean momentum operator $\hat{\pi}$, corresponding to the classical conserved quantity 'momentum') and non-relativistic boosts (the position operator $\hat{\kappa}$, corresponding to the classical conserved quantity 'initial position'). They are, explicitly,

$$
\begin{aligned}
& \hat{\pi}=-i \hbar \frac{\partial}{\partial \kappa} \\
& \hat{\kappa}=\kappa+\frac{i \hbar}{m} \tau \frac{\partial}{\partial \kappa},
\end{aligned}
$$

that is, those basic, canonically commuting operators with constant expectation values, that respect the solutions of the free Schrödinger equation, have constant matrix elements (and constant expectation values in particular) and fall down to well defined, time-independent operators in the Hilbert space of the free particle $L^{2}(\mathbb{R})$.

In general, these properties are satisfied whenever an operator $\hat{O}(t)$ can be written as

$$
\hat{O}(t)=\hat{U}\left(t, t_{0}\right) \hat{O} \hat{U}^{\dagger}\left(t, t_{0}\right),
$$

where $\hat{O}$ is $\hat{O}\left(t_{0}\right)$ and $\hat{U}\left(t, t_{0}\right)$ is the evolution operator satisfying the Schrödinger equation 4 . If the Hamiltonian is time-independent, as in the free particle case, time-evolution is a oneparameter group and then $\hat{U}\left(\tau, \tau_{0}\right)=\hat{U}\left(\tau-\tau_{0}\right)$.

Defining a generic Schrödinger equation operator, $\hat{S} \equiv i \hbar \frac{\partial}{\partial t}-\hat{H}$, taking $t_{0}=0(\hat{U}(t) \equiv$ $\hat{U}(t, 0))$ and reminding that $\hat{U}\left(t, t_{0}\right)^{\dagger} \hat{U}\left(t, t_{0}\right)=1$, it is clear that, for operators of the form (19):

$$
\begin{aligned}
& \hat{S} \hat{O}(t)|\psi(t)\rangle=\hat{S} \hat{U}(t) \hat{O}|\psi\rangle \equiv \hat{S} \hat{U}(t)\left|\psi^{\prime}\right\rangle=\hat{S}\left|\psi^{\prime}(t)\right\rangle=0 \\
& \frac{\partial}{\partial t}\langle\chi(t)|\hat{O}(t)| \psi(t)\rangle=0 \\
& \frac{\partial}{\partial t}\left(\hat{U}(t)^{\dagger} \hat{O}(t) \hat{U}(t)\right)=0 \\
& \frac{\mathrm{d}}{\mathrm{d} t} \hat{O}(t) \equiv \frac{\partial}{\partial t} \hat{O}(t)+\frac{i}{\hbar}[\hat{H}(t), \hat{O}(t)]=0
\end{aligned}
$$

(where $|\psi(t)\rangle \equiv \hat{U}(t)|\psi\rangle$ ), stating that those operators respect solutions, have constant matrix elements, fall down to define time-independent operators on the Hilbert space and are integers of the motion, respectively. (17) and (18) can be "de-evolved", so that

$$
\hat{U}(\tau)^{\dagger} \hat{\pi} \hat{U}(\tau)=-i \hbar \frac{\partial}{\partial \kappa}, \quad \hat{U}(\tau)^{\dagger} \hat{\kappa} \hat{U}(\tau)=\kappa
$$

\footnotetext{
${ }^{4}$ Note that $\hat{O}(t)$ is not the usual Heisenberg picture version $\hat{O}_{H}(t)=\hat{U}\left(t, t_{0}\right)^{\dagger} \hat{O} \hat{U}\left(t, t_{0}\right)$ of its associated operator in Schrödinger picture $\hat{O}$, although their relation is very simple when the Hamiltonian is time-independent.
} 
thus showing the properties above for the free particle.

What we are doing is to focus on these integrals of motion, $\hat{\pi}$ and $\hat{\kappa}$, so that the new operators position $\hat{X}$ and momentum $\hat{P}$ acting on $\mathcal{H}_{t}$ are also integrals of motion in the non-free system. These will be the generators of the basic symmetry in the non-free system. Dodonov and Man'ko [7] obtained these operators in particular cases by direct calculation, imposing them to commute with the Schrödinger equation $\hat{S}$. The difference is that, having related this system with that of the free particle, now it is clear how far one can go: the Schrödinger group and its enveloping algebra. Even more, the approach followed in [7] is only able to provide the basic operators (corresponding to linear functions in the classical solution manifold), since the condition of commuting with $\hat{S},[\hat{S}, \hat{O}(t)]=0$, is more restrictive than that considered here of respecting solutions, which is equivalent to $[\hat{S}, \hat{O}(t)] \sim \hat{S}$.

Let us apply the QAT (13) to (17) and (18). For a given operator $\hat{\pi}$ acting on $\mathcal{H}_{\tau}^{G}$ there is a corresponding operator $\hat{P}=\hat{A}^{-1} \hat{\pi} \hat{A}$ on $\mathcal{H}_{t}$. The action on functions $\phi(x, t)$ can then be obtained as follows:

$$
\begin{aligned}
\hat{P} \phi(x, t) & =\hat{A}^{-1} \hat{\pi} \hat{A} \phi(x, t)=\hat{A}^{-1} \hat{\pi} A^{*}\left(\sqrt{u_{2}} e^{-\frac{i}{2} \frac{m}{\hbar} \frac{1}{W} \frac{\dot{u}_{2}}{u_{2}} x^{2}} \phi(x, t)\right)= \\
& =\frac{1}{\sqrt{u_{2}}} e^{+\frac{i}{2} \frac{m}{\hbar} \frac{1}{W} \frac{\dot{u}_{2}}{u_{2}} x^{2}} A^{*-1}\left(-i \hbar \frac{\partial}{\partial \kappa} A^{*}\left(\sqrt{u_{2}} e^{-\frac{i}{2} \frac{m}{\hbar} \frac{1}{W} \frac{\dot{u}_{2}}{u_{2}} x^{2}} \phi(x, t)\right)\right)= \\
& =\frac{1}{\sqrt{u_{2}}} e^{+\frac{i}{2} \frac{m}{\hbar} \frac{1}{W} \frac{\dot{u}_{2}}{u_{2}} x^{2}}\left(-i \hbar u_{2} \frac{\partial}{\partial x}\left(\sqrt{u_{2}} e^{-\frac{i}{2} \frac{m}{\hbar} \frac{1}{W} \frac{\dot{u}_{2}}{u_{2}} x^{2}} \phi(x, t)\right)\right)= \\
& =\left(-i \hbar u_{2} \frac{\partial}{\partial x}-m \frac{\dot{u}_{2}}{W} x\right) \phi(x, t) .
\end{aligned}
$$

We can perform the same computation for the position operator and then we have:

$$
\begin{aligned}
& \hat{P}=-i \hbar u_{2} \frac{\partial}{\partial x}-m x \frac{\dot{u}_{2}}{W} \\
& \hat{X}=\frac{\dot{u}_{1}}{W} x+\frac{i \hbar}{m} u_{1} \frac{\partial}{\partial x},
\end{aligned}
$$

thus providing the generators of the realization of the (centrally-extended) Heisenberg-Weyl symmetry on the physical system corresponding to a general LSODE.

The properties of the operators (26) and (27), i.e. preserving solutions of (12), having constant matrix elements, falling to the Hilbert space, are ensured by the properties of (17) and (18) before the transformation, and will be explicitly checked for some particular cases in next Section. It will also become clear that the identity of both operators is preserved after the transformation.

Apart from $\hat{P}$ and $\hat{X}$, we can compute $\hat{P}^{2}, \hat{X}^{2}$ and $\hat{X P} \equiv \frac{1}{2}(\hat{X} \hat{P}+\hat{P} \hat{X})$ :

$$
\begin{aligned}
\hat{P}^{2} & =-\hbar^{2} u_{2}^{2} \frac{\partial^{2}}{\partial x^{2}}+i \hbar \frac{2 m u_{2} \dot{u}_{2}}{W} x \frac{\partial}{\partial x}+m^{2} \frac{\dot{u}_{2}^{2}}{W^{2}} x^{2}+i \hbar \frac{m u_{2} \dot{u}_{2}}{W} \\
\hat{X}^{2} & =\frac{\dot{u}_{1}^{2}}{W^{2}} x^{2}+i \hbar \frac{2 u_{1} \dot{u}_{1}}{m W} x \frac{\partial}{\partial x}-\hbar^{2} \frac{u_{1}^{2}}{m^{2}} \frac{\partial^{2}}{\partial x^{2}}+i \hbar \frac{u_{1} \dot{u}_{1}}{m W} \\
\hat{X P} & =\frac{\hbar^{2}}{m} u_{1} u_{2} \frac{\partial^{2}}{\partial x^{2}}-i \hbar \frac{\dot{u}_{1} u_{2}+u_{1} \dot{u}_{2}}{W} x \frac{\partial}{\partial x}-m \frac{\dot{u}_{1} \dot{u}_{2}}{W^{2}} x^{2}-i \hbar \frac{\dot{u}_{1} u_{2}+u_{1} \dot{u}_{2}}{2 W} .
\end{aligned}
$$

Their first-order versions, valid on solutions of (12), are:

$$
\begin{aligned}
\hat{P}^{2} & =i \hbar \frac{2 m u_{2}^{2}}{W} \frac{\partial}{\partial t}+i \hbar \frac{2 m u_{2} \dot{u}_{2}}{W} x \frac{\partial}{\partial x}+m^{2} \frac{\dot{u}_{2}^{2}-\omega^{2} u_{2}^{2}}{W^{2}} x^{2}+i \hbar \frac{m u_{2} \dot{u}_{2}}{W} \\
\hat{X}^{2} & =\frac{\dot{u}_{1}^{2}-\omega^{2} u_{1}^{2}}{W^{2}} x^{2}+i \hbar \frac{2 u_{1} \dot{u}_{1}}{m W} x \frac{\partial}{\partial x}+i \hbar \frac{2 u_{1}^{2}}{m W} \frac{\partial}{\partial t}+i \hbar \frac{u_{1} \dot{u}_{1}}{m W} \\
\hat{X P} & =-i \hbar \frac{2 u_{1} u_{2}}{W} \frac{\partial}{\partial t}-i \hbar \frac{\dot{u}_{1} u_{2}+u_{1} \dot{u}_{2}}{W} x \frac{\partial}{\partial x}-m \frac{\dot{u}_{1} \dot{u}_{2}-\omega^{2} u_{1} u_{2}}{W^{2}} x^{2}-i \hbar \frac{\dot{u}_{1} u_{2}+u_{1} \dot{u}_{2}}{2 W},
\end{aligned}
$$


which, together with $\hat{X}$ and $\hat{P}$, close the whole Schrödinger Lie algebra:

$$
\begin{aligned}
& {[\hat{X}, \hat{P}]=i \hbar} \\
& {\left[\hat{X}, \hat{P}^{2}\right]=2 i \hbar \hat{P} \quad\left[\hat{X}, \hat{X}^{2}\right]=0} \\
& {\left[\hat{P}, \hat{P}^{2}\right]=0 \quad\left[\hat{P}, \hat{X}^{2}\right]=-2 i \hbar \hat{X} \quad[\hat{P}, \hat{X P}]=-i \hbar \hat{P}} \\
& {\left[\hat{X}^{2}, \hat{P}^{2}\right]=4 i \hbar \hat{X P} \quad\left[\hat{X}^{2}, \hat{X P}\right]=2 i \hbar \hat{X}^{2} \quad\left[\hat{P}^{2}, \hat{X P}\right]=-2 i \hbar \hat{P}^{2} \text {. }}
\end{aligned}
$$

All these operators are well-defined on the solution space of the time-dependent Schrödinger equation, so that the action of one of them on a solution is again a solution. However, it is important to note once again that the Hamiltonian operator corresponding to the LSODE, that is, the quantum version of (11) $(\Lambda=0), \hat{H}$, although being a second order differential operator, can not be expressed in terms of these operators in general and then it does not close a Lie algebra with them. But that which is worse, it is not even a well-defined operator on the space of solutions of the Schrödinger equation, $\mathcal{H}_{t}$. As a consequence, $\hat{H}$ is not the generator of a one parameter group corresponding to conventional time evolution.

Instead, any linear combination of $\hat{P}^{2}, \hat{X}^{2}$ and $\hat{X P}$, say $\hat{\tilde{H}}$, can be adopted as a well-defined, Hermitian evolution-like generator. It has an associated eigenvalue equation and real spectrum, and its eigenvalues can be used to label solutions of (12) $(\Lambda=0)$ as its eigenfunctions. The particular choice of $\hat{\tilde{H}}$ to be taken depends purely on convenience and, for example, the similarity with the form of $\hat{H}$ of the particular physical system.

We would like to point out that there is an essential difference between the approach followed in [20] and ours. The reason is that the un-extended Schrödinger group is considered there as the fundamental symmetry of the damped harmonic oscillator, the origin of which is the analysis of the classical equations of motion in [4]. The approach based on QAT provides directly a representation of a central extension of the Schrödinger group adapted to the specific LSODE-type system 5. For the relevance of central extensions in Quantum Mechanics, we refer to [18, 21, 22, 23, 24, 25].

Let us stress that QAT can be useful to quickly perform some calculations, avoiding tedious, direct evaluations which can become extremely involved in the system under study. For example, it can be used to compute the quantum propagator for any LSODE-type quantum system, following the idea of Takagi in [13] for the simple case of the harmonic oscillator, or even the evolution operator $\hat{U}(t)$, which becomes very difficult to evaluate exactly when the Hamiltonian is time-dependent and does not commute with itself at different times.

Actually, the evolution operator of a LSODE system can be related with the free evolution operator. Having in mind the diagram (6), we write:

$$
\hat{A}(\hat{U}(t) \phi(x))=\hat{U}_{G}(\tau) \varphi(\kappa) .
$$

Here $\phi$ and $\varphi$ are the same function of only one $\operatorname{argument}(\kappa$ or $x)$ and we will denote $\varphi=\phi=\psi$. Then,

$$
\begin{aligned}
\hat{U}(t) \psi(x)=\hat{A}^{-1}\left(\hat{U}_{G}(\tau) \psi(\kappa)\right)=\frac{1}{\sqrt{u_{2}}} e^{\frac{i}{2} \frac{m}{\hbar} \frac{1}{W} \frac{\dot{u}_{2}}{u_{2}} x^{2}} A^{*-1}\left(\hat{U}_{G}(\tau) \psi(\kappa)\right)= \\
=\frac{1}{\sqrt{u_{2}}} e^{\frac{i}{2} \frac{m}{\hbar} \frac{1}{W} \frac{\dot{u}_{2}}{u_{2}} x^{2}} A^{*-1}\left(\hat{U}_{G}(\tau)\right) A^{*-1}(\psi(\kappa)) .
\end{aligned}
$$

\footnotetext{
${ }^{5}$ This fact is of the greatest relevance for the analysis of the inclusion of time-symmetry in [19] for the damped harmonic oscillator.
} 
To factorize the function $\psi$ and single out the general action of $\hat{U}(t)$, we compute

$$
A^{*-1}(\psi(\kappa))=\psi\left(\frac{x}{u_{2}}\right)=e^{\log \left(1 / u_{2}\right) x \frac{\partial}{\partial x}} \psi(x),
$$

where $e^{\log \left(1 / u_{2}\right) x \frac{\partial}{\partial x}}$ is a dilation operator which is not unitary. To unitarize this operator, the generator must be shifted from $x \frac{\partial}{\partial x}$ to $x \frac{\partial}{\partial x}+\frac{1}{2}$, so that the true unitary operator is then

$$
\hat{U}_{D}\left(\frac{1}{u_{2}}\right)=e^{\log \left(1 / u_{2}\right)\left(x \frac{\partial}{\partial x}+\frac{1}{2}\right)}=\frac{1}{\sqrt{u_{2}}} e^{\log \left(1 / u_{2}\right) x \frac{\partial}{\partial x}} .
$$

But the factor $\frac{1}{\sqrt{u_{2}}}$ is already present in the previous expression of $\hat{U}(t)$. Therefore, it now reads

$$
\begin{aligned}
\hat{U}(t)=e^{\frac{i}{2} \frac{m}{\hbar} \frac{1}{W} \frac{\dot{u}_{2}}{u_{2}} x^{2}} A^{*-1}\left(\hat{U}_{G}(\tau)\right) \hat{U}_{D}\left(\frac{1}{u_{2}}\right)= \\
=\frac{1}{\sqrt{u_{2}}} e^{\frac{i}{2} \frac{m}{\hbar} \frac{1}{W} \frac{\dot{u}_{2}}{u_{2}} x^{2}} e^{\frac{i \hbar}{2 m} u_{1} u_{2} \frac{\partial^{2}}{\partial x^{2}} e^{\log \left(1 / u_{2}\right) x \frac{\partial}{\partial x}} .}
\end{aligned}
$$

Its inverse is given by

$$
\hat{U}(t)^{-1}=\hat{U}(t)^{\dagger}=\sqrt{u_{2}} e^{\log \left(u_{2}\right) x \frac{\partial}{\partial x}} e^{\frac{-i \hbar}{2 m} u_{1} u_{2} \frac{\partial^{2}}{\partial x^{2}}} e^{-\frac{i}{2} \frac{m}{\hbar} \frac{1}{W} \frac{u_{2}}{u_{2}} x^{2}} .
$$

Interestingly, we have been able to obtain an exact expression for the evolution operator as a product of operators. No perturbative approximation method, which could become cumbersome in some cases, is needed for any LSODE-related quantum system to obtain the evolution operator. These results hold for $\Lambda \neq 0$ (see Appendix $\mathrm{A}$ ).

As a general comment before proceeding with the computation of the wave functions, let us go back to the relevance of conditions (14). Those have been chosen to preserve the identity of the variables and basic operators. Any other choice of solutions satisfying different initial conditions at any given initial time would have implications which must be kept under control. This was not taken into account in [7, which might result in some confusing derivations. A general shift

$$
u_{1} \rightarrow a u_{1}+b u_{2}, \quad u_{2} \rightarrow c u_{1}+d u_{2},
$$

with the condition $a d-b c=1$ to preserve the value of the Wronskian $W$, is equivalent to the canonical transformation in the basic operators

$$
\hat{X} \rightarrow a \hat{X}-\frac{b}{m} \hat{P}, \quad \hat{P} \rightarrow-c m \hat{X}+d \hat{P} .
$$

That is, the freedom in the choice of the solutions $u_{1}$ and $u_{2}$, which is a $S L(2, \mathbb{R})$ transformation, stands for a $S p(1, \mathbb{R})$ transformation in basic operators and defines a family of quantum Arnold transformations. Then, the relation between Hilbert spaces in the lower part of the diagram (6) turns into a non-trivial transformation:

$$
\hat{A}_{0} \psi(x) \equiv \hat{1} \psi(x) \rightarrow \sqrt{d} e^{-\frac{i c m x^{2}}{2 \hbar d}} \psi\left(\frac{x}{d}\right) .
$$

Following the general ideas already noted, any linear combination of quadratic operators belonging to the realization of the Schrödinger group (any operator in the subalgebra of (31)(33) ) can be chosen in such a way that its eigenfunctions solve (12). A specific combination of the operators (31)-(33) with constant coefficients $\tilde{\omega}$ and $\tilde{\gamma}$ was already chosen in [20] 6 :

$$
\hat{H}^{*}=\frac{1}{2 m} \hat{P}^{2}+\frac{1}{2} m \tilde{\omega}^{2} \hat{X}^{2}+\frac{\tilde{\gamma}}{2} \hat{X P} .
$$

\footnotetext{
${ }^{6}$ For the damped harmonic oscillator with constant $\omega$ and $\gamma$, coinciding with $\tilde{\omega}$ and $\tilde{\gamma}$ resp., this operator is the only one from the $S L(2, \mathbb{R})$ Schrödinger subalgebra which commutes with the Hamiltonian.
} 
The eigenfunctions of this operator, solutions of the Schrödinger equation, are

$$
\begin{aligned}
& \phi_{\nu}(x, t)=\frac{1}{\sqrt{\sqrt{2 \pi} \Gamma(\nu+1) \sqrt{\left(u_{2}-\tilde{\gamma} u_{1} / 2\right)^{2}+\tilde{\Omega}^{2} u_{1}^{2}}}} e^{\frac{i}{2 \hbar} m x^{2}\left(\frac{\tilde{\Omega}^{2} u_{1} /\left(u_{2}-\tilde{\gamma} u_{1} / 2\right)}{\left(u_{2}-\tilde{\gamma} u_{1} / 2\right)^{2}+\tilde{\Omega}^{2} u_{1}^{2}}+\frac{\dot{u}_{2}-\tilde{\gamma} \dot{u}_{1} / 2}{\left(u_{2}-\tilde{\gamma} u_{1} / 2\right) W}\right)} \\
& \left(\frac{u_{2}-\tilde{\gamma} u_{1} / 2-i \tilde{\Omega} u_{1}}{\sqrt{\left(u_{2}-\tilde{\gamma} u_{1} / 2\right)^{2}+\tilde{\Omega}^{2} u_{1}^{2}}}\right)^{\nu+\frac{1}{2}}\left(C_{1} D_{\nu}\left(\frac{\sqrt{\frac{2 m \tilde{\Omega}}{\hbar}} x}{\sqrt{\left(u_{2}-\tilde{\gamma} u_{1} / 2\right)^{2}+\tilde{\Omega}^{2} u_{1}^{2}}}\right)+C_{2} D_{-1-\nu}\left(\frac{i \sqrt{\frac{2 m \tilde{\Omega}}{\hbar}} x}{\sqrt{\left(u_{2}-\tilde{\gamma} u_{1} / 2\right)^{2}+\tilde{\Omega}^{2} u_{1}^{2}}}\right)\right),
\end{aligned}
$$

where $C_{1}$ and $C_{2}$ are arbitrary constants, $D_{\nu}$ are the parabolic cylinder functions [28], $\Gamma$ is the Gamma function, $\tilde{\Omega}=\sqrt{\tilde{\omega}^{2}-\frac{\tilde{\gamma}^{2}}{4}}$ and $\nu$ is in general a complex number.

In writing $\phi_{\nu}(x, t)$ we have kept the generality of the quantum Arnold transformation so that these solutions are valid for any LSODE-type system (the corresponding formula for a LSODE with a external force $\Lambda \neq 0$ is given in the Appendix $\mathrm{A}$. This family of wave functions is more general than the one found by Dodonov and Man'ko in [7] in that it contains a set of functions valid when $\frac{\tilde{\gamma}}{2}>\tilde{\omega}$ even for a general LSODE system. The associated spectrum of $\hat{H}^{*}$ is

$$
h^{*}=\hbar \tilde{\Omega}\left(\nu+\frac{1}{2}\right) \text {. }
$$

To obtain these solutions, we have taken advantage of the QAT itself performing the following steps. We solve the time-dependent Schrödinger equation corresponding to a harmonic oscillator with frequency $\tilde{\Omega}$, considering both the attractive and the repulsive cases, so that we obtain solutions in terms of parabolic cylinder functions. Then, we take the QAT from this "intermediate" system to the free one. We compose this QAT with the inverse QAT corresponding to passing the free system to the present LSODE system, obtaining this way solutions to the LSODE system Schrödinger equation, which are eigenfunctions of $\hat{H}_{H O \Omega}=\frac{1}{2 m} \hat{P}^{2}+\frac{1}{2} m \tilde{\Omega}^{2} \hat{X}^{2}$. Finally, making use of the freedom in the choice in $u_{1}$ and $u_{2}$, we perform the shift

$$
u_{1} \rightarrow u_{1}, \quad u_{2} \rightarrow u_{2}-\frac{\tilde{\gamma}}{2} u_{1} \quad \Rightarrow \quad \tilde{X} \rightarrow \hat{X}, \quad \tilde{P} \rightarrow \hat{P}+m \frac{\tilde{\gamma}}{2} \hat{X} .
$$

Its effect on the quadratic operators causes the expression of the particular combination

$$
\hat{H}_{H O \Omega}=\frac{1}{2 m} \hat{P}^{2}+\frac{1}{2} m \tilde{\Omega}^{2} \hat{X}^{2} \quad \rightarrow \quad \frac{1}{2 m} \hat{P}^{2}+\frac{1}{2} m \tilde{\omega}^{2} \hat{X}^{2}+\frac{\tilde{\gamma}}{2} \hat{X P}=\hat{H}^{*}
$$

to change. As a consequence, the obtained solutions turn into $\phi_{\nu}(x, t)$.

The condition of normalizability must be imposed to retain the physically valid solutions. And we observe that the normalizability of the wave functions depends on the specific values of $\tilde{\omega}$ and $\tilde{\gamma}$ in the expression (47), 7 . For $\tilde{\omega}>\frac{\tilde{\gamma}}{2}$, the normalizable solutions correspond to $C_{2}=0$ and $\nu=n$ an integer. These functions are written then in terms of the Hermite polynomials 8 . In the case when $\tilde{\Omega}$ is imaginary, the solutions are Dirac-delta normalizable for $\nu=-\frac{1}{2}+i \lambda$, with $\lambda$ a real number. The operator $\hat{H}^{*}$ shows a continuous, real, doubly degenerate spectrum in this case [29] (see also [7] for constant $\omega$ and $\gamma$ in the overdamping regime). The critical case $\tilde{\Omega}=0$ can be obtained as a limit of this case.

It must be emphasized then that the choice of these constants encodes the choice of the particular (arbitrary) quadratic operator belonging to the Schrödinger algebra used to label the solutions. In the framework of the quantum Arnold transformation, this freedom leads to other families of solutions, different from the one presented here.

\footnotetext{
${ }^{7}$ In the specific case of the damped harmonic oscillator, with constant $\omega$ and $\gamma$, it is possible to identify $\tilde{\omega} \equiv \omega$ and $\tilde{\gamma} \equiv \gamma(\tilde{\Omega} \equiv \Omega)$, so that the admissible wave functions vary depending on the regime.

${ }^{8}$ These are the eigenfunctions of the operator $\hat{H}^{*}$ (there denoted as $\hat{K}(t)$ ) found in [7] in the general LSODE case. It should be noted that they avoid, in this general case, the explicit mention of dimensional constants equivalent to $\tilde{\omega}$ and $\tilde{\gamma}$ and implicitly entrust the selection of the specific $\hat{K}(t)$ to the choice of the classical solutions, which might become rather confusing.
} 


\section{Dissipative systems: Hamiltonian vs. Hermitian operators}

Let us now have a close look at a couple of simple particular cases, extensively studied in the literature: the damped particle and the damped harmonic oscillator. Analogously, it is possible to analyze the harmonic oscillator from the QAT point of view. Although [13] and [7] contain some aspects of this analysis, it is possible to go a bit further and arrive at interesting results [26].

\subsection{Damped particle}

For $f=\gamma t, \omega=0$ and $\Lambda=0$ in (10), a Lagrangian for the damped particle can be given:

$$
L_{D P}=\frac{1}{2} m e^{\gamma t} \dot{x}^{2}
$$

where $\gamma$ is the damping constant. The equation of motion is then

$$
\ddot{x}+\gamma \dot{x}=0 .
$$

Two independent solutions for this equation, satisfying initial conditions (14),

$$
u_{1}(t)=\frac{1-e^{-\gamma t}}{\gamma}, \quad u_{2}(t)=1, \quad W(t)=e^{-\gamma t},
$$

provide the Arnold transformation for this system:

$$
\left\{\begin{array}{l}
\tau=\frac{1-e^{-\gamma t}}{\gamma} \\
\kappa=x \\
\varphi=\phi
\end{array}\right.
$$

which turns out to be simply a reparametrization in time.

The Schrödinger equation takes the form

$$
i \hbar \frac{\partial \phi}{\partial t}=\hat{H}_{D P} \phi \equiv-\frac{\hbar^{2}}{2 m} e^{-\gamma t} \frac{\partial^{2} \phi}{\partial x^{2}},
$$

and the corresponding basic symmetry generators

$$
\hat{P}=-i \hbar \frac{\partial}{\partial x}, \quad \hat{X}=x+\frac{i \hbar}{m \gamma}\left(1-e^{-\gamma t}\right) \frac{\partial}{\partial x} .
$$

The crucial point is to realize that, in fact, the Hamiltonian operator $\hat{H}_{D P}$ does not make sense as an operator acting on the space of solutions of (56), while $\hat{P}$ and $\hat{X}$ do. This can be checked by direct computation. For a given solution $\phi$, the equation satisfied by $\phi^{\prime} \equiv \hat{H}_{D P} \phi$ is no longer (56), the reason being that $\hat{H}_{D P}$ does not commute with the Schrödinger equation, while $\hat{X} \phi$, for instance, does solve it:

$$
\left(i \hbar \frac{\partial}{\partial t}+\frac{\hbar^{2}}{2 m} e^{-\gamma t} \frac{\partial^{2}}{\partial x^{2}}\right)(\hat{X} \phi)=\left(x+\frac{i \hbar}{m \gamma}\left(1-e^{-\gamma t}\right) \frac{\partial}{\partial x}\right)\left(i \hbar \frac{\partial \phi}{\partial t}+\frac{\hbar^{2}}{2 m} e^{-\gamma t} \frac{\partial^{2} \phi}{\partial x^{2}}\right)=0,
$$

showing that the Schrödinger equation and $\hat{X}$ do commute.

There is yet another way to check explicitly that $\hat{H}_{D P}$ is ill-defined in the quotient space by the time-evolution generated by itself. Formally, the equation (56) can be solved defining a time-evolution operator $\hat{U}\left(t, t_{0}\right)$. The fact that $\hat{H}_{D P}$ commutes at different times,

$$
\left[\hat{H}_{D P}\left(t_{1}\right), \hat{H}_{D P}\left(t_{2}\right)\right]=0
$$


makes the calculation of $\hat{U}\left(t, t_{0}\right)$ and its action on other operators simple:

$$
\hat{U}\left(t, t_{0}\right)=e^{\frac{-i}{\hbar} \int_{t_{0}}^{t} \hat{H}_{D P}\left(t^{\prime}\right) \mathrm{dt}^{\prime}}=e^{\frac{i \hbar}{2 m \gamma}\left(e^{-\gamma t_{0}}-e^{-\gamma t}\right) \frac{\partial^{2}}{\partial x^{2}}} .
$$

If we choose $t_{0}=0$, in agreement with conditions (14) imposed on solutions (154), we recover the Arnold-transformed free evolution operator, as could be expected. However, the computation of the evolution operator directly and the possibility of obtaining it using QAT is not in any way trivial when (59) does not hold.

By means of the action of $\hat{U}(t, 0) \equiv \hat{U}(t)$ on the basic operators $\hat{P}$ and $\hat{X}$, or loosely speaking, using $\hat{U}(t)$ to "de-evolve" them until time $t_{0}=0$, we can show that they match the form (19) and determine their action on wave functions depending only on $x$. This action, in this simple case, can be computed by expanding the exponential evolution operator and performing the commutation operations at each order of the expansion, leading to

$$
\hat{U}^{\dagger}(t) \hat{x} \hat{U}(t)=x, \quad \hat{U}^{\dagger}(t) \hat{p} \hat{U}(t)=-i \hbar \frac{\partial}{\partial x},
$$

which do not depend on time $t$ and take the usual Galilei form 9 . This automatically guarantees the three properties mentioned in the previous Section. In contrast, $\hat{H}_{D P}$ does not come down to the quotient by the time evolution generated by itself:

$$
\hat{U}^{\dagger}(t) \hat{H}_{D P} \hat{U}(t)=-\frac{\hbar^{2}}{2 m} e^{-\gamma t} \frac{\partial^{2}}{\partial x^{2}} .
$$

The reason for $\hat{X}$ and $\hat{P}$ to have good properties is that they are mapped from the free, basic symmetry generators (18) and (17) by the Arnold transformation (so that they are also symmetry generators of the damped particle system), while $\hat{H}_{D P}$ is not. It is then natural to map one of the quadratic operators belonging to the Schrödinger algebra of the free particle to make it act on the space of quantum solutions of the damped particle. This evolution-like operator defines a proper eigenvalue problem, that can be used to find solutions for (56). We could choose the operator $\hat{H}^{*}$ already mentioned, but in this simple case we prefer to illustrate another rather natural possibility: the free Galilean Hamiltonian

$$
\hat{H}_{G} \equiv \frac{\hat{P}^{2}}{2 m}=-\frac{\hbar^{2}}{2 m} \frac{\partial^{2}}{\partial x^{2}} .
$$

In fact, the practical approach would be to solve the free, time-independent Schrödinger equation and Arnold-transform the solutions to obtain (non-stationary) solutions for (56). For example, plane waves are mapped into:

$$
\phi_{k}(x, t)=e^{i k x-i \frac{\hbar k^{2}}{2 m \gamma}\left(1-e^{-\gamma t}\right)} .
$$

The observations made above for the damped particle, being quite trivial, can help to clarify the general case.

\subsection{Damped harmonic oscillator}

Let us consider a friction function linear in time $f=\gamma t$, as in the case of the damped particle, but also a non-zero constant frequency $\omega$ and no external force. The Lagrangian for the Caldirola-Kanai system reads

$$
L_{D H O}=e^{\gamma t}\left(\frac{1}{2} m \dot{x}^{2}-\frac{1}{2} m \omega^{2} x^{2}\right) .
$$

\footnotetext{
${ }^{9}$ Note that the "de-evolved" operators take the form of those in (57) when $t=0$. But in general, the correct way to take the quotient by time evolution is that shown in (61).
} 
The classical equation of motion is then

$$
\ddot{x}+\gamma \dot{x}+\omega^{2} x=0 .
$$

Two independent solutions of (6ㅏ), satisfying convenient initial conditions (14) are

$$
u_{1}(t)=\frac{1}{\Omega} e^{-\frac{\gamma}{2} t} \sin \Omega t, \quad u_{2}(t)=e^{-\frac{\gamma}{2} t} \cos \Omega t+\frac{\gamma}{2 \Omega} e^{-\frac{\gamma}{2} t} \sin \Omega t,
$$

where again $W(t) \equiv \dot{u}_{1}(t) u_{2}(t)-u_{1}(t) \dot{u}_{2}(t)=e^{-\gamma t}$, and

$$
\Omega=\sqrt{\omega^{2}-\frac{\gamma^{2}}{4}}
$$

Note that these solutions have good limit in the case of critical damping $\omega=\frac{\gamma}{2}$.

Particularizing the quantum Arnold transformation (13) for the free Schrödinger equation (77), the Caldirola-Kanai equation is obtained:

$$
i \hbar \frac{\partial \phi}{\partial t}=\hat{H}_{D H O} \phi \equiv-\frac{\hbar^{2}}{2 m} e^{-\gamma t} \frac{\partial^{2} \phi}{\partial x^{2}}+\frac{1}{2} m \omega^{2} x^{2} e^{\gamma t} \phi .
$$

Basic quantum operators are now given by

$$
\begin{aligned}
& \hat{P}=-i \hbar \frac{e^{-\frac{\gamma t}{2}}}{2 \Omega}(2 \Omega \cos \Omega t+\gamma \sin \Omega t) \frac{\partial}{\partial x}+m \frac{e^{\frac{\gamma t}{2}}}{4 \Omega}\left(\gamma^{2}+4 \Omega^{2}\right) \sin \Omega t x, \\
& \hat{X}=\frac{e^{\frac{\gamma t}{2}}}{2 \Omega}(2 \Omega \cos \Omega t-\gamma \sin \Omega t) x+i \hbar \frac{e^{-\frac{\gamma t}{2}}}{m \Omega} \sin \Omega t \frac{\partial}{\partial x} .
\end{aligned}
$$

It is worth to note that these operators match those that were already found in [7] by hand, in looking for integrals of motion.

Again, the key observation is that $\hat{H}_{D H O}$ does not make sense as an operator acting on the space of solutions of (69), while $\hat{P}$ and $\hat{X}$ do respect solutions. Although this can be proved by direct calculation, it is more instructive to obtain the evolution operator $\hat{U}\left(t, t_{0}\right)$. But the fact that $\hat{H}_{D H O}$ does not commute at different times $\left[\hat{H}_{D H O}\left(t_{1}\right), \hat{H}_{D H O}\left(t_{2}\right)\right] \neq 0$ makes its calculation trickier using conventional methods, as already mentioned.

Generically, one would approach the problem resorting to a perturbative method. An appropriate method to solve the operator equation for $\hat{U}(t) \equiv \hat{U}(t, 0)$ corresponding to (69) (we now make explicit the time dependence of the Hamiltonian),

$$
i \hbar \frac{\partial}{\partial t} \hat{U}(t)=\hat{H}_{D H O}(t) \hat{U}(t),
$$

is that of the Magnus expansion [27] (see Appendix B).

However, it is a good idea to take advantage of the QAT instead. The explicit expression for the exact evolution operator encountered for the damped harmonic oscillator is rather involved and is found substituting (67) in (42):

$$
\begin{aligned}
\hat{U}(t)=\sqrt{\frac{2 \Omega e^{\frac{\gamma}{2} t}}{2 \Omega \cos \Omega t+\gamma \sin \Omega t}} & e^{\frac{i}{2} \frac{m}{\hbar} \frac{-2 \omega^{2} e^{\gamma t} \sin \Omega t}{2 \Omega \cos \Omega t+\gamma \sin \Omega t} x^{2}} \\
& e^{\frac{i \hbar}{2 m} \frac{1}{2 \Omega^{2}} e^{-\gamma t} \sin \Omega t(2 \Omega \cos \Omega t+\gamma \sin \Omega t) \frac{\partial^{2}}{\partial x^{2}}} e^{\log \left(\frac{2 \Omega e^{\frac{\gamma}{2}} t}{2 \Omega \cos \Omega t+\gamma \sin \Omega t}\right) x \frac{\partial}{\partial x}} .
\end{aligned}
$$


$\hat{U}^{\dagger}(t)$ is obtained analogously. It is then possible to check the de-evolution of the operators simply expanding the exponentials to the desired order, just as one would do for the free evolution operator. This allows to state that:

$$
\hat{U}^{\dagger}(t) \hat{X} \hat{U}(t)=x, \quad \hat{U}^{\dagger}(t) \hat{P} \hat{U}(t)=-i \hbar \frac{\partial}{\partial x},
$$

as expected.

The action of $\hat{U}(t)$ on $\hat{H}_{D H O}(t)$ shows that it does not fall down to the quotient by the time evolution generated by itself. In fact, the de-evolution,

$$
\begin{aligned}
& \hat{U}^{\dagger}(t) \hat{H}_{D H O} \hat{U}(t)= \\
&\left(-\frac{\hbar^{2}}{2 m} \frac{\partial^{2}}{\partial x^{2}}+\frac{1}{2} m \omega^{2} x^{2}\right)-\gamma t\left(-\frac{\hbar^{2}}{2 m} \frac{\partial^{2}}{\partial x^{2}}-\frac{1}{2} m \omega^{2} x^{2}\right) \\
& \quad+\frac{\gamma^{2} t^{2}}{2}\left(-\frac{\hbar^{2}}{2 m} \frac{\partial^{2}}{\partial x^{2}}+\frac{1}{2} m \omega^{2} x^{2}+\frac{2 \omega^{2}}{\gamma}\left(-i \hbar x \frac{\partial}{\partial x}-i \hbar\right)\right)+\mathcal{O}\left(t^{3}\right),
\end{aligned}
$$

does depend on time at any order in the time expansion.

We remark once again that there is no actual need for a perturbative method to obtain $\hat{U}(t)$ in this case when QAT is used.

The computation of the wave functions, solutions of (69), can follow the steps shown in Section 3. We select the operator $\hat{H}^{*}$ particularized for the damped harmonic oscillator, that is, substituting $u_{1}$ and $u_{2}$ by (67) and identifying $\tilde{\omega} \equiv \omega$ and $\tilde{\gamma} \equiv \gamma$. Its general eigenfunctions are given by the corresponding expression (48), and the spectrum (49) of $\hat{H}^{*}$ will depend on the regime fixed by the specific value of $\Omega$.

\section{Conclusions}

The analysis carried out in this paper permits to deal with the quantum theory of any LSODE-type dynamical system, using known properties of the quantum free particle. The quantum Arnold transformation provides basic operators and establishes that the symmetry group of the free particle, the Schrödinger group, can be transferred to a realization on the LSODE system. This result turns out to be of practical use when performing some computations, for instance finding solutions of the Schrödinger equation or the evolution operator, especially when the Hamiltonian does not commute with itself at different times. Even in these cases it is possible to give exact expressions, obtained in a non-perturbative manner. It is noteworthy that these calculations lead to the knowledge of objects in the quantum theory with the only requirement that the classical solutions of the LSODE are known.

In a way, Arnold transformation allows to interpret LSODE-type forces, including dissipation linear in velocity, as effects observed in a "non-inertial reference frame". The use of the present scheme goes beyond the study of the simple damped harmonic oscillator, finding applicability in quite different branches of physics, such as Cosmology, where a scalar field appears (inflaton) satisfying equations in time which can be read as a LSODE. In this respect we are preparing the study of the specific example of the harmonic oscillator with time-dependent frequency [30].

It is worth mentioning that the Arnold transformation used in this work is just a particular case of a broader class of transformations which link free particle equations to even classical non-linear equations. Research in this direction would potentially lead to extremely useful and interesting results. We believe that a good starting point for this purpose was presented in [17], although some effort to establish the explicit connection with the free particle would be in order. 
Let us end up with a final general comment concerning the symmetry under time translation of the quantum system associated with a LSODE. Even though it is possible to set up a clear framework to deal with any LSODE-type quantum system by employing the quantum Arnold transformation, it does not provide by itself a well-defined operator associated with proper (true) time evolution. The reason is that conventional time evolution is not included in general in the symmetry group that can be imported from the free system: the Hamiltonian does not belong to the specific representation of the Schrödinger algebra. One may wonder what happens if time evolution symmetry is required. We pursue this interesting issue at least for the damped harmonic oscillator in [19].

\section{A Appendix: Inhomogeneous LSODE}

We give here the general Arnold transformation with an extra external force term $\Lambda$ and the corresponding generalization of the main results above. This computation follows analogous steps as those shown before.

The general QAT is given by

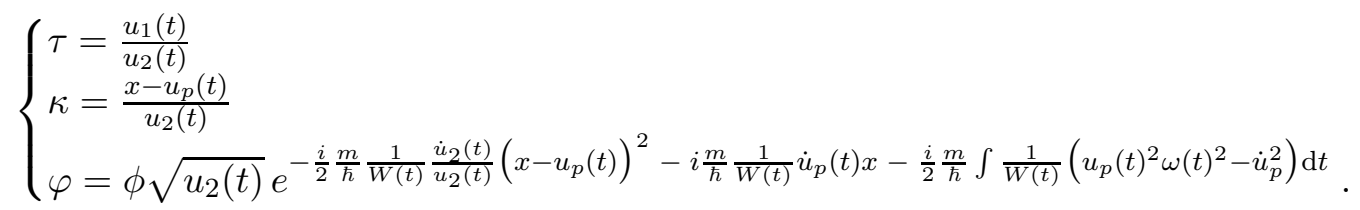

The extra conditions to be imposed on the classical solution $u_{p}(t)$ to preserve the identity of $x$ and $t$ before and after the transformation are:

$$
u_{p}(0)=0, \quad \dot{u}_{p}(0)=0 .
$$

In fact, the solution $u_{p}(t)$ can be expressed:

$$
u_{p}(t)=K_{1}(t) u_{1}(t)+K_{2}(t) u_{2}(t)
$$

where:

$$
K_{1}(t)=\int_{0}^{t} \frac{u_{2}\left(t^{\prime}\right)}{W\left(t^{\prime}\right)} \Lambda\left(t^{\prime}\right) \mathrm{d} t^{\prime}, \quad K_{2}(t)=-\int_{0}^{t} \frac{u_{1}\left(t^{\prime}\right)}{W\left(t^{\prime}\right)} \Lambda\left(t^{\prime}\right) \mathrm{d} t^{\prime},
$$

The transformation (76) leads to the expressions for basic operators:

$$
\begin{aligned}
& \hat{P}=-i \hbar u_{2} \frac{\partial}{\partial x}-m \frac{\dot{u}_{2}}{W}\left(x-u_{p}\right)-m \frac{u_{2}}{W} \dot{u}_{p} \\
& \hat{X}=\frac{\dot{u}_{1}}{W}\left(x-u_{p}\right)+\frac{u_{1}}{W} \dot{u}_{p}+\frac{i \hbar}{m} u_{1} \frac{\partial}{\partial x} .
\end{aligned}
$$

The evolution operator reads

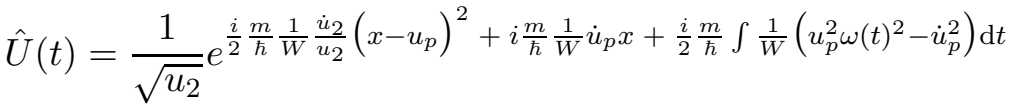

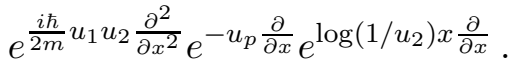


Finally, the general solution of the corresponding Schrödinger equation, eigenfunction of the operator $\hat{H}^{*}$, is:

$$
\begin{aligned}
& \phi_{\nu}(x, t)=\frac{1}{\sqrt{\sqrt{2 \pi} \Gamma(\nu+1) \sqrt{\left(u_{2}-\tilde{\gamma} u_{1} / 2\right)^{2}+\tilde{\Omega}^{2} u_{1}^{2}}}} e^{\left(\frac{i}{2 \hbar} m\left(x-u_{p}\right)^{2} \frac{\left.\tilde{\Omega}^{2} u_{1} / u_{2}-\tilde{\gamma} u_{1} / 2\right)}{\left(u_{2}-\tilde{\gamma} u_{1} / 2\right)^{2}+\tilde{\Omega}^{2} u_{1}^{2}}+\frac{i}{2 \hbar} m x^{2} \frac{u_{2}-\tilde{\gamma} \dot{u}_{1} / 2}{\left(u_{2}-\tilde{\gamma} u_{1} / 2\right) W}\right)} \\
& e^{\left(\frac{i m}{2 \hbar} \int \frac{1}{W}\left(u_{p}^{2} \omega(t)^{2}-\dot{u}_{p}^{2}\right) \mathrm{d} t-\frac{i m x u_{p}\left(\dot{u}_{2}-\tilde{\tilde{r}} \dot{u}_{1} / 2\right)}{\hbar W\left(u_{2}-\tilde{\gamma} u_{1} / 2\right)}+\frac{i m u_{p}^{2}\left(\dot{u}_{2}-\tilde{\gamma} \dot{u}_{1} / 2\right)}{2 \hbar W\left(u_{2}-\tilde{\gamma} u_{1} / 2\right)}+\frac{i m x \dot{u}_{p}}{\hbar W}\right)} \\
& \left(\frac{u_{2}-\tilde{\gamma} u_{1} / 2-i \tilde{\Omega} u_{1}}{\sqrt{\left(u_{2}-\tilde{\gamma} u_{1} / 2\right)^{2}+\tilde{\Omega}^{2} u_{1}^{2}}}\right)^{\nu+\frac{1}{2}}\left(C_{1} D_{\nu}\left(\frac{\sqrt{\frac{2 m \tilde{\Omega}}{\hbar}}}{\sqrt{\left(u_{2}-\tilde{\gamma} u_{1} / 2\right)^{2}+\tilde{\Omega}^{2} u_{1}^{2}}}\right)+C_{2} D_{-1-\nu}\left(\frac{i \sqrt{\frac{2 m \tilde{\Omega}}{\hbar}}}{\sqrt{\left(u_{2}-\tilde{\gamma} u_{1} / 2\right)^{2}+\tilde{\Omega}^{2} u_{1}^{2}}}\right)\right) .
\end{aligned}
$$

\section{B Appendix: The Magnus expansion}

The Magnus expansion was introduced as a tool to solve non-autonomous linear differential equations for linear operators and has the very attractive property of leading to approximate solutions which exhibit unitarity at any order of approximation. This is in contrast to the representation in terms of the time-ordering operator $\mathcal{T}$ introduced by Dyson.

A solution to (72) is given by

$$
\hat{U}(t)=e^{\hat{\Omega}(t)}, \quad \hat{\Omega}(0)=0,
$$

and a series expansion for the matrix in the exponent

$$
\hat{\Omega}(t)=\sum_{k=1}^{\infty} \hat{\Omega}_{k}(t)
$$

which is called the Magnus expansion. We can write down the first three terms of that series:

$$
\begin{aligned}
& \hat{\Omega}_{1}(t)=\int_{0}^{t} \mathrm{~d} t_{1}\left(-\frac{i}{\hbar} \hat{H}_{D H O}\left(t_{1}\right)\right) \\
& \hat{\Omega}_{2}(t)=\frac{1}{2} \int_{0}^{t} \mathrm{~d} t_{1} \int_{0}^{t_{1}} \mathrm{~d} t_{2}\left[-\frac{i}{\hbar} \hat{H}_{D H O}\left(t_{1}\right),-\frac{i}{\hbar} \hat{H}_{D H O}\left(t_{2}\right)\right] \\
& \hat{\Omega}_{3}(t)=\frac{1}{6} \int_{0}^{t} \mathrm{~d} t_{1} \int_{0}^{t_{1}} \mathrm{~d} t_{2} \int_{0}^{t_{2}} \mathrm{~d} t_{3}\left(\left[-\frac{i}{\hbar} \hat{H}_{D H O}\left(t_{1}\right),\left[-\frac{i}{\hbar} \hat{H}_{D H O}\left(t_{2}\right),-\frac{i}{\hbar} \hat{H}_{D H O}\left(t_{3}\right)\right]\right]\right. \\
&\left.+\left[-\frac{i}{\hbar} \hat{H}_{D H O}\left(t_{3}\right),\left[-\frac{i}{\hbar} \hat{H}_{D H O}\left(t_{2}\right),-\frac{i}{\hbar} \hat{H}_{D H O}\left(t_{1}\right)\right]\right]\right)
\end{aligned}
$$

However, a good iterative method to obtain the operator $\hat{\Omega}(t)$ is given by the formula:

$$
\begin{aligned}
\hat{\Omega}^{[n]}(t) & =\sum_{k=0}^{\infty} \frac{B_{k}}{k !} \int_{0}^{t} \mathrm{~d} t_{1} \operatorname{ad}_{\hat{\Omega}^{[\mathrm{n}-1]}\left(\mathrm{t}_{1}\right)}^{\mathrm{k}}\left(-\frac{\mathrm{i}}{\hbar} \hat{\mathrm{H}}_{\mathrm{DHO}}\left(\mathrm{t}_{1}\right)\right) \\
\hat{\Omega}(t) & =\lim _{n \rightarrow \infty} \hat{\Omega}^{[n]}(t),
\end{aligned}
$$

where $B_{k}$ are the Bernoulli numbers and

$$
\operatorname{ad}_{\hat{\mathrm{A}}}^{0}(\hat{\mathrm{B}}) \equiv \hat{\mathrm{B}}, \quad \operatorname{ad}_{\hat{\mathrm{A}}}^{1}(\hat{\mathrm{B}}) \equiv[\hat{\mathrm{A}}, \hat{\mathrm{B}}], \quad \operatorname{ad}_{\hat{\mathrm{A}}}^{\mathrm{k}}(\hat{\mathrm{B}}) \equiv\left[\operatorname{ad}_{\hat{\mathrm{A}}}^{\mathrm{k}-1}(\hat{\mathrm{B}}), \hat{\mathrm{B}}\right] .
$$


We have computed the operator $\hat{\Omega}(t)$ for the case of the damped harmonic oscillator to sixth order of approximation, to give:

$$
\begin{aligned}
& \hat{\Omega}^{[6]}(t)= \\
& -\frac{i}{\hbar} t\left(\left(1+\frac{\gamma^{2} t^{2}}{6}+\frac{\gamma^{4} t^{4}}{120}\left(1+\frac{2 \omega^{2}}{\gamma^{2}}\right)+\frac{\gamma^{6} t^{6}}{5040}\left(1+\frac{16 \omega^{2}}{\gamma^{2}}+\frac{32 \omega^{4}}{3 \gamma^{4}}\right)\right)\left(-\frac{\hbar^{2}}{2 m} \frac{\partial^{2}}{\partial x^{2}}+\frac{1}{2} m \omega^{2} x^{2}\right)\right. \\
& -\frac{\gamma t}{2}\left(1+\frac{\gamma^{2} t^{2}}{12}+\frac{\gamma^{4} t^{4}}{360}\left(1+\frac{6 \omega^{2}}{\gamma^{2}}\right)\right)\left(-\frac{\hbar^{2}}{2 m} \frac{\partial^{2}}{\partial x^{2}}-\frac{1}{2} m \omega^{2} x^{2}\right) \\
& \left.\quad+\frac{\gamma \omega^{2} t}{6}\left(1+\frac{\gamma^{2} t^{2}}{20}\left(1+\frac{4 \omega^{2}}{3 \gamma^{2}}\right)+\frac{\gamma^{4} t^{4}}{840}\left(1+\frac{44 \omega^{2}}{3 \gamma^{2}}+\frac{16 \omega^{4}}{3 \gamma^{4}}\right)\right)\left(-i \hbar x \frac{\partial}{\partial x}-\frac{1}{2} i \hbar\right)\right)
\end{aligned}
$$

With this approximation to $\hat{\Omega}(t)$, obtained by means of (88), one can obtain the quotient by time evolution of a certain operator $\hat{O}(t)$, given by:

$$
\hat{O}=e^{-\hat{\Omega}(t)} \hat{O}(t) e^{\hat{\Omega}(t)}=\sum_{k=0}^{\infty} \frac{1}{k !} \operatorname{ad}_{-\hat{\Omega}(\mathrm{t})}^{\mathrm{k}}(\hat{\mathrm{O}}(\mathrm{t})) .
$$

These formulas lead to the same results found in Subsection 4.2 .

\section{Acknowledgments}

Work partially supported by the Fundación Séneca, Spanish MICINN and Junta de Andalucía under projects 08814/PI/08, FIS2008-06078-C03-01 and FQM219-FQM1951, respectively.

The authors wish to thank M. Calixto for useful discussions and comments.

\section{References}

[1] Caldirola P., Nuovo Cimento 18, 393 (1941)

[2] Kanai E. Prog. Theor. Phys. 3, 440 (1948)

[3] Martini R. and Kersten P.H.M., J. Phys. A 16, 455 (1983)

[4] Cerveró J.M. and Villarroel J., J. Phys. A 17, 1777 (1984)

[5] Niederer U., Helv. Phys. Acta 45, 802 (1972); 46, 191 (1973); 47, 167 (1974)

[6] Hioe F.T., J. Math. Phys. 15, 445 (1974); Yuen H.P., Phys. Rev. A 13, 2226 (1976).

[7] Dodonov V.V. and Man'ko V.I., Phys. Rev. A 20, 550 (1979)

[8] Brittin W.E., Phys. Rev. 77, 396 (1950)

[9] Razavy M., Classical and Quantum Dissipative Systems (Imperial College Press, 2005).

[10] Schuch D., Phys. Rev. A 55, 935 (1997)

[11] Arnold V.I., Geometrical methods in the theoy of ordinary differential equations (Springer, 1998)

[12] Lutzky M., J. Phys. A 11, 249 (1978) 
[13] Takagi S., Prog. Theor. Phys. 84, 1019 (1990)

[14] Huang M-C., Wu M-C., Chin. J. Phys. 36, 566 (1998)

[15] Kanasugi H. and Okada H., Prog. Theor. Phys. 93, 949 (1995)

[16] Mostafazadeh A., Phys. Rev. A 55, 4084 (1997)

[17] Mostafazadeh A., J. Phys. A 31, 6495 (1998)

[18] Aldaya V., Navarro-Salas J., Bisquert J. and Loll R., J. Math. Phys. 33, 3087 (1992)

[19] Guerrero J., Aldaya V., Cossío F. and López-Ruiz F.F., A round trip from Caldirola to Bateman in GROUP 28: The XXVIII ICGTMP, Newcastle 26-30 July 2010, to appear in IoP Journal of Physics Conference Series;

Aldaya V., Cossío F., Guerrero J. and López-Ruiz F.F., A symmetry trip from Caldirola to Bateman damped systems, to be published.

[20] Cerveró J.M. and Villarroel J., J. Phys. A 17, 2963 (1984)

[21] Bargmann V., Ann. Math. 59, 1 (1954)

[22] Lévy-Leblond J.M., Commun. Math. Phys. 12, 64 (1969)

[23] Aldaya V. and de Azcárraga J.A., J. Math. Phys. 23, 1297 (1982)

[24] Aldaya V. and Guerrero J. Rep. Math. Phys. 47, 213 (2001)

[25] Aldaya V., Calixto M., Guerrero J. and López-Ruiz F.F., J. Nonl. Math. Phys. 15, 1 (2008)

[26] Guerrero J., López-Ruiz F.F., Aldaya V. and Cossío F., arXiv:1010.5525

[27] Blanes S., Casas F. and Oteo J.A., Ros J., Phys. Rep. 470, 151 (2009)

[28] Gradshteyn I.S. and Ryzhik I.M., Table of integrals, series and products (Academic, 2007)

[29] Aldaya V., de Azcárraga J.A. and Wolf K.B., J. Math. Phys. 25, 506 (1984)

[30] In preparation. 